\title{
Highly Soluble [1]Benzothieno[3,2-b]benzothiophene (BTBT) Derivatives for High-Performance, Solution-Processed Organic Field-Effect Transistors
}

Hideaki Ebata, Takafumi Izawa, Eigo Miyazaki, Kazuo Takimiya, Masaaki Ikeda, Hirokazu Kuwabara, and Tatsuto Yui

\section{Contents}

1. Synthesis

2. Physicochemical properties

3. Solubility and stability

4. Single crystal X-ray analysis of $\mathrm{C}_{12}$-BTBT

5. Device fabrications and evaluations

6. XRDs of $\mathrm{C}_{n}$-BTBT thin film

7. In-plane XRD (GIXRD) of $\mathrm{C}_{12}$-BTBT thin film

8. References

\section{Synthesis}

General: All chemicals and solvents are of reagent grade unless otherwise indicated. Palladium on charcoal $(10 \% \mathrm{Pd} / \mathrm{C})$ was purchased from Aldrich and used as received. Several solvents including diisopropyl amine, benzene, and toluene were purified by standard distillation procedures prior to use. $\quad[1]$ Benzothieno[3,2-b][1]benzothiophene ${ }^{\mathrm{S} 1}$ and 2,7-diiode[1]benzothieno[3,2-b][1] benzothiophene ${ }^{\mathrm{S} 2}$ were synthesized according to the reported procedures. Melting points were uncorrected. Nuclear magnetic resonance spectra were obtained in deuterated chloroform with a JEOL Lambda 400 spectrometer operating at $400 \mathrm{MHz}$ for ${ }^{1} \mathrm{H}$ or $100 \mathrm{MHz}$ for ${ }^{13} \mathrm{C}$ with TMS as internal reference; chemical shifts $(\delta)$ are reported in parts per

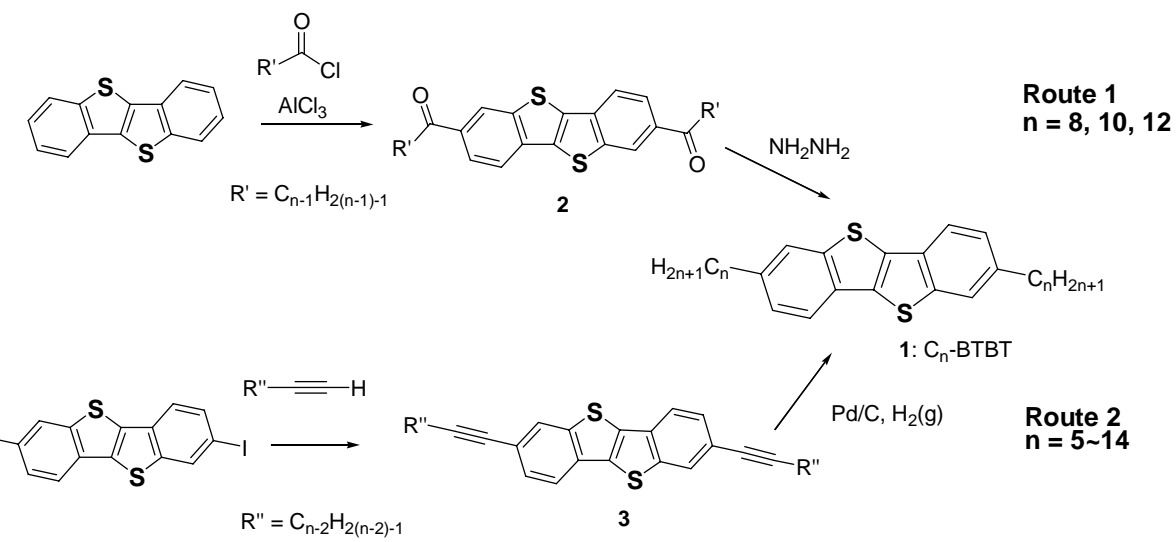

Scheme 1. Synthesis of $\mathrm{C}_{n}$-BTBTs: two different approaches were examined. 
million. EI-MS spectra were obtained on a Shimadzu QP-5050A spectrometer using an electron impact ionization procedure $(70 \mathrm{eV})$. The molecular ion peaks of the sulfur-containing compounds showed a typical isotopic pattern, and all the mass peaks are reported based on ${ }^{32} \mathrm{~S}$.

\section{Route 1}

General procedure for the Friedel-Crafts acylation on the parent [1]benzothieno[3,2-b][1]benzothiophene ${ }^{\mathrm{S} 1}$

Caution! This procedure involves use of acid chloride and aluminum chloride, which fume in the presence of moisture liberating hydrochloric acid. Therefore, this must be carried out in a well-ventilated fume hood with disposable gloves. Into a solution of [1]benzothieno[3,2-b][1]benzothiophene $(1.0 \mathrm{~g}, 4.2 \mathrm{mmol})$ in dichloromethane $(100 \mathrm{~mL})$ was added aluminum chloride $(3.0 \mathrm{~g}, 23 \mathrm{mmol})$ at $-10{ }^{\circ} \mathrm{C}$, and the solution was cooled to $-78^{\circ} \mathrm{C}$. Then, acid chloride $(21 \mathrm{mmol})$ was added dropwise, and the mixture was stirred for $1 \mathrm{~h}$ at the same temperature. The reaction mixture was allowed to stand without cooling and stirred for $48 \mathrm{~h}$ at $\mathrm{rt}$. The mixture was diluted with water to give a precipitate. The precipitate was collected by filtration and washed consecutively with water and methanol. The crude solid was purified by recrystallization from toluene to give 2,7-bis(acyl)BTBT (2) as colorless crystals.

([1]benzothienopheno[3,2-b]benzothienophene-2,7-diyl)bis(octan-1-one) $\left(2, \mathbf{R}^{\prime}=\mathbf{C}_{7} \mathbf{H}_{15}\right)$ :

$67 \%$ yield. mp $250-251{ }^{\circ} \mathrm{C} ;{ }^{1} \mathrm{H}$ NMR $\delta 8.58(\mathrm{~d}, J=1.2 \mathrm{~Hz}, 2 \mathrm{H}), 8.09$ (dd, $\left.J=8.0,1.2 \mathrm{~Hz}, 2 \mathrm{H}\right)$, $7.99(\mathrm{~d}, J=8.0 \mathrm{~Hz}, 2 \mathrm{H}), 3.09(\mathrm{t}, J=7.2 \mathrm{~Hz}, 4 \mathrm{H}), 1.75-1.85(\mathrm{~m}, 4 \mathrm{H}), 1.26-1.48(\mathrm{~m}, 16 \mathrm{H}), 0.90(\mathrm{t}, J$ $=7.2 \mathrm{~Hz}, 6 \mathrm{H})$; $\mathrm{MS}(\mathrm{EI}) \mathrm{m} / \mathrm{z} 492\left(\mathrm{M}^{+}\right)$.

([1]benzothienopheno[3,2-b]benzothienophene-2,7-diyl)bis(decan-1-one) (2, R' = $\left.\mathbf{C}_{9} \mathbf{H}_{19}\right)$ : $60 \%$ yield. mp $241-242{ }^{\circ} \mathrm{C} ;{ }^{1} \mathrm{H}$ NMR $\delta 8.58(\mathrm{~d}, J=1.2 \mathrm{~Hz}, 2 \mathrm{H}), 8.09(\mathrm{dd}, J=8.0,1.2 \mathrm{~Hz}, 2 \mathrm{H})$, 7.99 (d, $J=8.0 \mathrm{~Hz}, 2 \mathrm{H}), 3.09$ (t, $J=7.2 \mathrm{~Hz}, 4 \mathrm{H}), 1.75-1.85$ (m, 4H), 1.20-1.48 (m, 24H), 0.89 (t, $J$ $=7.2 \mathrm{~Hz}, 6 \mathrm{H})$; $\mathrm{MS}(\mathrm{EI}) \mathrm{m} / \mathrm{z} 548\left(\mathrm{M}^{+}\right)$.

([1]benzothienopheno[3,2-b]benzothienophene-2,7-diyl)bis(dodecan-1-one) (2, $\left.\mathbf{R}^{\prime}=\mathbf{C}_{\mathbf{1 1}} \mathbf{H}_{\mathbf{2} 3}\right)$ : $44 \%$ yield. mp $220-221{ }^{\circ} \mathrm{C} ;{ }^{1} \mathrm{H}$ NMR $\delta 8.58(\mathrm{~d}, J=1.2 \mathrm{~Hz}, 2 \mathrm{H}), 8.09(\mathrm{dd}, J=8.0,1.2 \mathrm{~Hz}, 2 \mathrm{H})$, $7.99(\mathrm{~d}, J=8.0 \mathrm{~Hz}, 2 \mathrm{H}), 3.08$ (t, $J=7.2 \mathrm{~Hz}, 4 \mathrm{H}), 1.75-1.85(\mathrm{~m}, 4 \mathrm{H}), 1.10-1.70(\mathrm{~m}, 32 \mathrm{H}), 0.89$ (t, $J=7.2 \mathrm{~Hz}, 6 \mathrm{H})$; MS (EI) $\mathrm{m} / \mathrm{z} 604\left(\mathrm{M}^{+}\right)$.

General procedure for the Wolf-Kishner reduction of 2,7-bis(acyl)[1]benzothieno[3,2-b][1]benzothiophene ${ }^{\mathrm{s} 1}$

Caution! This procedure involves use of hydrazine hydrate, which is a violent poison and causes delayed eye irritation. Therefore, this must be carried out in a well-ventilated fume hood with disposable gloves and protective glasses. A mixture of 2,7-bis(acyl)BTBT (2, 2.0 mmol), potassium hydroxide $(614 \mathrm{mg}, 11 \mathrm{mmol})$, hydrazine hydrate $(6.1 \mathrm{~mL}, 51 \mathrm{mmol})$ in diethylene glycol $(92 \mathrm{~mL})$ was heated to $100{ }^{\circ} \mathrm{C}$ for $1 \mathrm{~h}$ and then further heated at $210{ }^{\circ} \mathrm{C}$ for $5 \mathrm{~h}$. The 
reaction mixture was cooled, and the resulting precipitate was collected by filtration, washed with water and methanol. Column chromatography of the crude product on silica gel eluted with hexane gave 2,7-dialkylBTBT $\left(\mathrm{R}_{\mathrm{f}}=0.6\right)$, which was further purified by recrystallization from hexane to give analytically pure samples as colorless microcrystals. Spectroscopic data for $\mathrm{C}_{8^{-}}, \mathrm{C}_{10^{-}}$, and $\mathrm{C}_{12}$-BTBT were identical with those obtained from the samples prepared with the route 2 (vide infra).

\section{Route 2}

General procedure for the palladium catalyzed Sonogashira coupling of 2,7-diiodo-BTBT with 1-alkynes.

1-Alkyne (2.7 mmol), $\mathrm{PdCl}_{2}\left(\mathrm{PPh}_{3}\right)_{2}(67 \mathrm{mg}, 0.1 \mathrm{mmol})$, and $\mathrm{CuI}$ (39 mg, $\left.0.2 \mathrm{mmol}\right)$ were added successively to a deaerated solution of 2,7-diiode[1]benzothieno[3,2-b][1]benzothiophene (492 mg, $1 \mathrm{mmol})$ in diisopropylamine $(15 \mathrm{~mL})$ and benzene $(15 \mathrm{~mL})$. The resulting mixture was stirred for $8 \mathrm{~h}$ at room temperature, then diluted with water $(30 \mathrm{~mL})$, and extracted with chloroform $(30 \mathrm{~mL} \times$ $3)$. The combined extract was washed with water $(100 \mathrm{~mL} \times 3)$ and dried $\left(\mathrm{MgSO}_{4}\right)$. Evaporation of the solvent gave a crude solid, which was subjected to column chromatography on silica gel eluted with dichloromethane-hexane $(1: 3, \quad \mathrm{v} / \mathrm{v})$ to give 2,7-dialkyn-1-yl[1]benzothieno[3,2-b][1]benzothiophene (3) as a colorless solid. Recrystallization of the solid from hexane gave analytical samples.

\section{2,7-Dipentyn-1-yl[1]benzothieno[3,2-b][1]benzothiophene $\left(3, \mathbf{R}\right.$ ” = $\left.\mathrm{C}_{3} \mathrm{H}_{7}\right)$ :}

$74 \%$ yield. mp $197-200{ }^{\circ} \mathrm{C} ;{ }^{1} \mathrm{H}$ NMR $\delta 7.95(\mathrm{~d}, J=1.2 \mathrm{~Hz}, 2 \mathrm{H}), 7.76(\mathrm{~d}, J=8.0 \mathrm{~Hz}, 2 \mathrm{H}), 7.47$ $(\mathrm{dd}, J=1.2,8.0 \mathrm{~Hz}, 2 \mathrm{H}), 2.44(\mathrm{t}, J=7.2 \mathrm{~Hz}, 4 \mathrm{H}), 1.67(\mathrm{~m}, 4 \mathrm{H}), 1.08(\mathrm{t}, J=7.6 \mathrm{~Hz}, 6 \mathrm{H}) ;{ }^{13} \mathrm{C} \mathrm{NMR}$ $\delta$ 142.2, 134.0, 132.0, 128.4, 126.9, 121.2, 120.9, 91.4, 80.6, 22.0, 21.5, 13.6; MS (EI) m/z 372 $\left(\mathrm{M}^{+}\right)$; Anal. Calcd for $\mathrm{C}_{24} \mathrm{H}_{20} \mathrm{~S}_{2}$ : C, 77.37; H, 5.41. Found: C, 77.06; H, 5.41.

2,7-Dihexyn-1-yl[1]benzothieno[3,2-b][1]benzothiophene (3, $\mathbf{R}$ ” $\left.=\mathbf{C}_{\mathbf{4}} \mathbf{H}_{\mathbf{9}}\right)$ :

$74 \%$ yield. mp $160-162{ }^{\circ} \mathrm{C} ;{ }^{1} \mathrm{H}$ NMR $\delta 7.92(\mathrm{~d}, J=1.2 \mathrm{~Hz}, 2 \mathrm{H}), 7.73(\mathrm{~d}, J=8.3 \mathrm{~Hz}, 2 \mathrm{H}), 7.45$ $(\mathrm{dd}, J=1.2,8.3 \mathrm{~Hz}, 2 \mathrm{H}), 2.46(\mathrm{t}, J=6.8 \mathrm{~Hz}, 4 \mathrm{H}), 1.66-1.59(\mathrm{~m}, 4 \mathrm{H}), 1.56-1.47(\mathrm{~m}, 4 \mathrm{H}), 0.97(\mathrm{t}, J$ $=7.3 \mathrm{~Hz}, 6 \mathrm{H}) ;{ }^{13} \mathrm{C}$ NMR $\delta 142.2,134.0,132.0,128.4,126.9,121.2,120.9,91.5,80.4,30.8,22.1$, 19.2, 13.7; MS (EI) m/z $400\left(\mathrm{M}^{+}\right)$; Anal. Calcd for $\mathrm{C}_{26} \mathrm{H}_{24} \mathrm{~S}_{2}$ : C, 77.95; H, 6.04. Found: C, 77.93; H, 6.01 .

2,7-Diheptyn-1-yl[1]benzothieno[3,2-b][1]benzothiophene (3, $\mathbf{R}$ ” = $\left.\mathbf{C}_{5} \mathbf{H}_{11}\right)$ :

$75 \%$ yield. mp $131-133{ }^{\circ} \mathrm{C} ;{ }^{1} \mathrm{H}$ NMR $\delta 7.94(\mathrm{~d}, J=1.6 \mathrm{~Hz}, 2 \mathrm{H}), 7.76(\mathrm{~d}, J=8.0 \mathrm{~Hz}, 2 \mathrm{H}), 7.47$ $(\mathrm{dd}, J=1.6,8.0 \mathrm{~Hz}, 2 \mathrm{H}), 2.45(\mathrm{t}, J=7.6 \mathrm{~Hz}, 4 \mathrm{H}), 1.70-1.60(\mathrm{~m}, 4 \mathrm{H}), 1.53-1.30(\mathrm{~m}, 8 \mathrm{H}), 0.94(\mathrm{t}, J$ $=7.2 \mathrm{~Hz}, 6 \mathrm{H}) ;{ }^{13} \mathrm{C} \mathrm{NMR} \delta 142.2,134.0,132.0,128.4,126.9,121.2,120.9,91.6,80.4,31.2,28.5$, 22.3, 19.5, 14.0 ; MS (EI) m/z $428\left(\mathrm{M}^{+}\right)$; Anal. Calcd for $\mathrm{C}_{28} \mathrm{H}_{28} \mathrm{~S}_{2}$ : C, 78.46; H, 6.58. Found: C, $78.30 ; \mathrm{H}, 6.60$.

2,7-Dioctyn-1-yl[1]benzothieno[3,2-b][1]benzothiophene (3, R” = $\left.\mathbf{C}_{6} \mathbf{H}_{13}\right)$ :

$77 \%$ yield. mp $119-121{ }^{\circ} \mathrm{C} ;{ }^{1} \mathrm{H}$ NMR $\delta 7.94(\mathrm{~s}, 2 \mathrm{H}), 7.76(\mathrm{~d}, J=8.2 \mathrm{~Hz}, 2 \mathrm{H}), 7.47$ (d, J=8.2 Hz, 
2H), $2.45(\mathrm{t}, J=7.1 \mathrm{~Hz}, 4 \mathrm{H}), 1.67-1.60(\mathrm{~m}, 4 \mathrm{H}), 1.52-1.44(\mathrm{~m}, 4 \mathrm{H}), 1.37-1.32(\mathrm{~m}, 8 \mathrm{H}), 0.92(\mathrm{t}, J$ $=6.8 \mathrm{~Hz}, 6 \mathrm{H}) ;{ }^{13} \mathrm{C} \mathrm{NMR} \delta 142.1,134.0,131.9,128.4,126.9,121.1,120.9,91.5,80.4,31.4,28.7$, 28.6, 22.6, 19.5, 14.1; MS (EI) $\mathrm{m} / \mathrm{z} 456\left(\mathrm{M}^{+}\right)$; Anal. Calcd for $\mathrm{C}_{30} \mathrm{H}_{32} \mathrm{~S}_{2}$ : C, 78.90; H, 7.06. Found: C, 79.03; H, 6.92.

2,7-Dinonyn-1-yl[1]benzothieno[3,2-b][1]benzothiophene (3, $\mathbf{R}$ ” = $\left.\mathbf{C}_{7} \mathbf{H}_{15}\right)$ :

$83 \%$ yield. mp $112-114{ }^{\circ} \mathrm{C} ;{ }^{1} \mathrm{H}$ NMR $\delta 7.94(\mathrm{~d}, J=1.2 \mathrm{~Hz}, 2 \mathrm{H}), 7.74(\mathrm{~d}, J=8.3 \mathrm{~Hz}, 2 \mathrm{H}), 7.47$ $(\mathrm{dd}, J=1.2,8.3 \mathrm{~Hz}, 2 \mathrm{H}), 2.44(\mathrm{t}, J=7.3 \mathrm{~Hz}, 4 \mathrm{H}), 1.67-1.60(\mathrm{~m}, 4 \mathrm{H}), 1.49-1.43(\mathrm{~m}, 4 \mathrm{H})$, $1.38-1.32(\mathrm{~m}, 12 \mathrm{H}), 0.90(\mathrm{t}, J=7.0 \mathrm{~Hz}, 6 \mathrm{H}) ;{ }^{13} \mathrm{C} \mathrm{NMR} \delta 142.2,134.0,132.0,128.4,126.9,121.2$, 121.0, 91.6, 80.4, 31.8, 28.94, 28.86, 28.76, 22.7, 19.5, 14.1; MS (EI) m/z $484\left(\mathrm{M}^{+}\right)$; Anal. Calcd for $\mathrm{C}_{32} \mathrm{H}_{36} \mathrm{~S}_{2}$ : C, 79.29; H, 7.49. Found: C, 79.36; H, 7.53.

2,7-Didecyn-1-yl[1]benzothieno[3,2-b][1]benzothiophene (3, R” = $\left.\mathbf{C}_{8} \mathbf{H}_{17}\right)$ :

$72 \%$ yield. mp $110-112{ }^{\circ} \mathrm{C} ;{ }^{1} \mathrm{H}$ NMR $\delta 7.94(\mathrm{~d}, J=1.2 \mathrm{~Hz}, 2 \mathrm{H}), 7.77(\mathrm{~d}, J=8.4 \mathrm{~Hz}, 2 \mathrm{H}), 7.47$ $(\mathrm{dd}, J=1.2,8.3 \mathrm{~Hz}, 2 \mathrm{H}), 2.45(\mathrm{t}, J=7.2 \mathrm{~Hz}, 4 \mathrm{H}), 1.66-1.60(\mathrm{~m}, 4 \mathrm{H}), 1.52-1.42(\mathrm{~m}, 4 \mathrm{H})$, $1.39-1.20(\mathrm{~m}, 16 \mathrm{H}), 0.90(\mathrm{t}, J=7.2 \mathrm{~Hz}, 6 \mathrm{H}) ;{ }^{13} \mathrm{C} \mathrm{NMR} \delta 142.2,134.0,132.0,128.4,126.9,121.2$, 121.0, 91.6, 80.4, 31.9, 29.22, 29.25, 29.0, 28.7, 22.7, 19.5, 14.1; MS (EI) m/z $512\left(\mathrm{M}^{+}\right)$; Anal. Calcd for $\mathrm{C}_{34} \mathrm{H}_{40} \mathrm{~S}_{2}$ : C, 79.63; H, 7.86. Found: C, 79.58; H, 7.78.

2,7-Diundecyn-1-yl[1]benzothieno[3,2-b][1]benzothiophene (3, R” = $\left.\mathbf{C}_{9} \mathbf{H}_{19}\right)$ :

$84 \%$ yield. mp 107-109 ${ }^{\circ} \mathrm{C} ;{ }^{1} \mathrm{H}$ NMR $\delta 7.93$ (s, 2H), 7.73 (d, J=8.4 Hz, 2H), 7.46 (d, J=8.4 Hz, 2H), 2.45 (t, $J=7.1 \mathrm{~Hz}, 4 \mathrm{H}), 1.67-1.60(\mathrm{~m}, 4 \mathrm{H}), 1.51-1.44(\mathrm{~m}, 4 \mathrm{H}), 1.39-1.29(\mathrm{~m}, 20 \mathrm{H}), 0.89(\mathrm{t}$, $J=6.8 \mathrm{~Hz}, 6 \mathrm{H}) ;{ }^{13} \mathrm{C}$ NMR $\delta 142.2,134.0,132.0,128.4,126.9,121.2,121.1,91.6,80.4,31.8,29.5$, 29.3, 29.2, 29.0, 28.7, 22.7, 19.5, 14.1; MS (EI) $\mathrm{m} / \mathrm{z} 540\left(\mathrm{M}^{+}\right)$; Anal. Calcd for $\mathrm{C}_{36} \mathrm{H}_{44} \mathrm{~S}_{2}$ : C, 79.94; H, 8.20. Found: C, 79.83; H, 8.16.

2,7-Didodecyn-1-yl[1]benzothieno[3,2-b][1]benzothiophene (3, R" = $\left.\mathbf{C}_{10} \mathbf{H}_{21}\right)$ :

$85 \%$ yield. mp 96-97 ${ }^{\circ} \mathrm{C} ;{ }^{1} \mathrm{H}$ NMR $\delta 7.93(\mathrm{~d}, J=1.4 \mathrm{~Hz}, 2 \mathrm{H}), 7.74(\mathrm{~d}, J=8.3 \mathrm{~Hz}, 2 \mathrm{H}), 7.46$ (dd, $J=1.4,8.3 \mathrm{~Hz}, 2 \mathrm{H}), 2.44(\mathrm{t}, J=7.1 \mathrm{~Hz}, 4 \mathrm{H}), 1.67-1.59(\mathrm{~m}, 4 \mathrm{H}), 1.51-1.43(\mathrm{~m}, 4 \mathrm{H}), 1.32-1.28(\mathrm{~m}$,

$24 \mathrm{H}), 0.88(\mathrm{t}, J=6.8 \mathrm{~Hz}, 6 \mathrm{H}) ;{ }^{13} \mathrm{C}$ NMR $\delta$ 142.2, 134.0, 132.0, 128.4, 126.9, 121.2, 121.0, 91.6, 80.4, 31.9, 29.61, 29.56, 29.3, 29.2, 29.0, 28.8, 22.7, 19.5, 14.1; MS (EI) m/z $568\left(\mathrm{M}^{+}\right)$; Anal. Calcd for Calcd for $\mathrm{C}_{38} \mathrm{H}_{48} \mathrm{~S}_{2}$ : C, 80.22; H, 8.50. Found: C, 80.12; H, 8.34.

2,7-Ditridecyn-1-yl[1]benzothieno[3,2-b][1]benzothiophene (3, R" = $\left.\mathbf{C}_{11} \mathbf{H}_{23}\right)$ :

$79 \%$ yield. mp 102-104 ${ }^{\circ} \mathrm{C} ;{ }^{1} \mathrm{H}$ NMR $\delta 7.93(\mathrm{~s}, 2 \mathrm{H}), 7.75(\mathrm{~d}, J=8.3 \mathrm{~Hz}, 2 \mathrm{H}), 7.46(\mathrm{~d}, J=8.3 \mathrm{~Hz}$, 2H), $2.45(\mathrm{t}, J=7.1 \mathrm{~Hz}, 4 \mathrm{H}), 1.67-1.60(\mathrm{~m}, 4 \mathrm{H}), 1.51-1.43(\mathrm{~m}, 4 \mathrm{H}), 1.32-1.27(\mathrm{~m}, 28 \mathrm{H}), 0.88(\mathrm{t}$, $J=7.0 \mathrm{~Hz}, 6 \mathrm{H}) ;{ }^{13} \mathrm{C} \mathrm{NMR} \delta 142.2,134.0,132.0,128.4,126.9,121.2,121.0,91.6,80.4,31.9$, 29.61, 29.56, 29.3, 29.2, 29.0, 28.8, 22.7, 19.5, 14.1; MS (EI) m/z $596\left(\mathrm{M}^{+}\right)$; Anal. Calcd for $\mathrm{C}_{40} \mathrm{H}_{52} \mathrm{~S}_{2}:$ C, 80.48; H, 8.78. Found: C, 80.39; H, 8.73.

2,7-Ditetradecyn-1-yl[1]benzothieno[3,2-b][1]benzothiophene (3, R' = $\left.\mathbf{C}_{\mathbf{1 2}} \mathbf{H}_{25}\right)$ :

$89 \%$ yield. mp $101-102{ }^{\circ} \mathrm{C} ;{ }^{1} \mathrm{H}$ NMR $\delta 7.93(\mathrm{~s}, J=1.2 \mathrm{~Hz}, 2 \mathrm{H}), 7.70(\mathrm{~d}, J=8.3 \mathrm{~Hz}, 2 \mathrm{H}), 7.46$ (dd, $J=1.2,8.3 \mathrm{~Hz}, 2 \mathrm{H}), 2.45(\mathrm{t}, J=7.0 \mathrm{~Hz}, 4 \mathrm{H}), 1.67-1.59(\mathrm{~m}, 4 \mathrm{H}), 1.51-1.43(\mathrm{~m}, 4 \mathrm{H}), 1.32-1.27(\mathrm{~m}$, $28 \mathrm{H}), 0.88(\mathrm{t}, J=7.0 \mathrm{~Hz}, 6 \mathrm{H}) ;{ }^{13} \mathrm{C} \mathrm{NMR} \delta 142.2,134.0,132.0,128.4,126.9,121.2,121.0,91.6$, $80.4,31.9,29.68,29.66(\times 2), 29.55,29.4,29.2,29.0,28.7,22.7,19.5,14.1 ; \mathrm{MS}(\mathrm{EI}) \mathrm{m} / \mathrm{z} 624$ 
$\left(\mathrm{M}^{+}\right)$; Anal. Calcd for $\mathrm{C}_{42} \mathrm{H}_{56} \mathrm{~S}_{2}: \mathrm{C}, 80.71 ; \mathrm{H}, 9.03$. Found: $\mathrm{C}, 80.72 ; \mathrm{H}, 9.01$.

General procedure for hydrogenation of 2,7-dialkenyl-BTBT in the presence of $\mathrm{Pd} / \mathrm{C}$ as a catalyst.

2,7-Dialkenyl-BTBT (3, $1 \mathrm{mmol}), 10 \% \mathrm{Pd} / \mathrm{C}(20 \mathrm{wt} \%$ based on the amount of substrate), and distilled toluene $(33 \mathrm{~mL})$ were placed in a $50 \mathrm{~mL}$ round bottomed flask, and the vessel was carefully vacuumed and then purged with hydrogen gas several times. The mixture was stirred at $\mathrm{rt}$, and the progress of hydrogenation was traced by TLC analysis. After the starting material disappeared on a TLC plate (usually it took more than $12 \mathrm{~h}$ ), the catalyst was filtered off, and the filtrate was concentrated. Column chromatography of the residue on silica gel eluted with hexane gave 2,7-dialkylBTBT $\left(\mathrm{R}_{\mathrm{f}}=0.6\right)$, which was further purified by recrystallization from hexane to give analytically pure samples as colorless microcrystals.

2,7-Dipentyl[1]benzothieno[3,2-b][1]benzothiophene ( $\mathbf{n}=5)$ :

$71 \%$ yield. mp $121-122{ }^{\circ} \mathrm{C} ;{ }^{1} \mathrm{H} \operatorname{NMR} \delta 7.77(\mathrm{~d}, J=8.0 \mathrm{~Hz}, 2 \mathrm{H}), 7.71(\mathrm{~s}, 2 \mathrm{H}), 7.28(\mathrm{~d}, J=8.0 \mathrm{~Hz}$, $2 \mathrm{H}), 2.76(\mathrm{t}, J=8.0 \mathrm{~Hz}, 4 \mathrm{H}), 1.75-1.65(\mathrm{~m}, 4 \mathrm{H}), 1.45-1.30(\mathrm{~m}, 8 \mathrm{H}), 0.92(\mathrm{t}, J=7.2 \mathrm{~Hz}, 6 \mathrm{H}) ;{ }^{13} \mathrm{C}$ NMR $\delta 142.4,140.0,132.5,131.2,125.8,123.3,121.1,36.1,31.5,31.4,22.6,14.1$; MS (EI) $m / z$ $380\left(\mathrm{M}^{+}\right)$; Anal. Calcd for ${ }_{24} \mathrm{H}_{28} \mathrm{~S}_{2}$ : C, 75.74; H, 7.42; H, 5.41. Found: C, 75.79; H, 7.46.

2,7-Dihexyl[1]benzothieno[3,2-b][1]benzothiophene $(\mathbf{n}=\mathbf{6})$ :

$88 \%$ yield. mp $122-124{ }^{\circ} \mathrm{C} ;{ }^{1} \mathrm{H}$ NMR $\delta 7.72(\mathrm{~d}, J=8.3 \mathrm{~Hz}, 2 \mathrm{H}), 7.67$ (s, 2H), 7.23 (d, $J=8.3 \mathrm{~Hz}$, 2H), $2.72(\mathrm{t}, J=7.8 \mathrm{~Hz}, 4 \mathrm{H}), 1.70-1.63(\mathrm{~m}, 4 \mathrm{H}), 1.37-1.31(\mathrm{~m}, 12 \mathrm{H}), 0.88(\mathrm{t}, J=5.8 \mathrm{~Hz}, 6 \mathrm{H}) ;{ }^{13} \mathrm{C}$ NMR $\delta 142.3,140.0,132.5,131.1,125.7,123.3,121.0,36.1,31.73,31.68,29.0,22.6,14.1$; MS (EI) $\mathrm{m} / \mathrm{z} 408\left(\mathrm{M}^{+}\right)$; Anal. Calcd for $\mathrm{C}_{26} \mathrm{H}_{32} \mathrm{~S}_{2}$ : C, 76.41; H, 7.89. Found: C, 76.43; H, 7.92.

2,7-Diheptyl[1]benzothieno[3,2-b][1]benzothiophene ( $\mathbf{n}=7)$ :

$88 \%$ yield. mp $129-132{ }^{\circ} \mathrm{C} ;{ }^{1} \mathrm{H}$ NMR $\delta 7.76(\mathrm{~d}, J=8.0 \mathrm{~Hz}, 2 \mathrm{H}), 7.71(\mathrm{~s}, 2 \mathrm{H}), 7.27(\mathrm{~d}, J=8.0 \mathrm{~Hz}$, 2H), $2.76(\mathrm{t}, J=7.6 \mathrm{~Hz}, 4 \mathrm{H}), 1.76-1.64(\mathrm{~m}, 4 \mathrm{H}), 1.45-1.20(\mathrm{~m}, 16 \mathrm{H}), 0.88(\mathrm{t}, J=7.2 \mathrm{~Hz}, 6 \mathrm{H}) ;{ }^{13} \mathrm{C}$ NMR $\delta 142.4,140.0,132.5,131.1,125.8,123.3,121.0,36.1,31.8,31.7,29.3,29.2,22.7,14.1$; MS (EI) $\mathrm{m} / \mathrm{z} 436\left(\mathrm{M}^{+}\right)$; Anal. Calcd for $\mathrm{C}_{28} \mathrm{H}_{36} \mathrm{~S}_{2}$ : C, 77.01; H, 8.31. Found: C, 77.09; H, 8.36.

2,7-Dioctyl[1]benzothieno[3,2-b][1]benzothiophene $(\mathbf{n}=\mathbf{8})$ : $82 \%$ yield. mp $129-131{ }^{\circ} \mathrm{C} ;{ }^{1} \mathrm{H}$ NMR $\delta 7.75(\mathrm{~d}, J=8.3 \mathrm{~Hz}, 2 \mathrm{H}), 7.69$ (s, 2H), 7.26 (d, $J=8.3 \mathrm{~Hz}$, 2H), $2.74(\mathrm{t}, J=7.7 \mathrm{~Hz}, 4 \mathrm{H}), 1.69(\mathrm{~m}, 4 \mathrm{H}), 1.34-1.27(\mathrm{~m}, 20 \mathrm{H}), 0.88(\mathrm{t}, J=6.7 \mathrm{~Hz}, 6 \mathrm{H}) ;{ }^{13} \mathrm{C}$ NMR $\delta 142.4,140.0,132.5,131.1,125.8,123.3,121.0,36.1,31.9,31.7,29.5,29.33,29.27,22.7$, 14.1; MS (EI) $\mathrm{m} / \mathrm{z} 464\left(\mathrm{M}^{+}\right)$; Anal. Calcd for $\mathrm{C}_{30} \mathrm{H}_{40} \mathrm{~S}_{2}$ : C, 77.53; H, 8.67. Found: C, 77.39; H, 8.67 .

2,7-Dinonyl[1]benzothieno[3,2-b][1]benzothiophene $(\mathbf{n}=\mathbf{9})$ :

91\% yield. mp $129-131{ }^{\circ} \mathrm{C} ;{ }^{1} \mathrm{H}$ NMR $\delta 7.72(\mathrm{~d}, J=8.3 \mathrm{~Hz}, 2 \mathrm{H}), 7.66(\mathrm{~s}, 2 \mathrm{H}), 7.22(\mathrm{~d}, J=8.3 \mathrm{~Hz}$, $2 \mathrm{H}), 2.71(\mathrm{t}, J=7.3 \mathrm{~Hz}, 4 \mathrm{H}), 1.62-1.69(\mathrm{~m}, 4 \mathrm{H}), 1.31-1.25(\mathrm{~m}, 24 \mathrm{H}), 0.87(\mathrm{t}, J=6.8 \mathrm{~Hz}, 6 \mathrm{H}) ;{ }^{13} \mathrm{C}$ NMR $\delta 142.3,140.0,132.5,131.1,125.8,123.3,121.0,36.1,31.9,31.7,29.57,29.55$ 29.3(×2), 22.7, 14.1; MS (EI) m/z $492\left(\mathrm{M}^{+}\right)$; Anal. Calcd for $\mathrm{C}_{32} \mathrm{H}_{44} \mathrm{~S}_{2}$ : C, 77.99; H, 9.00. Found: C, 78.04; H, 
8.98 .

2,7-Didecyl[1]benzothieno[3,2-b][1]benzothiophene (n = 10):

$88 \%$ yield. mp $124-126{ }^{\circ} \mathrm{C} ;{ }^{1} \mathrm{H}$ NMR $\delta 7.76(\mathrm{~d}, J=7.6 \mathrm{~Hz}, 2 \mathrm{H}), 7.70$ (s, 2H), 7.27 (d, J= 7.6 Hz,

2H), $2.75(\mathrm{t}, J=7.6 \mathrm{~Hz}, 4 \mathrm{H}), 1.73-1.64(\mathrm{~m}, 4 \mathrm{H}), 1.41-1.29(\mathrm{~m}, 28 \mathrm{H}), 0.89(\mathrm{t}, J=7.6 \mathrm{~Hz}, 6 \mathrm{H}) ;{ }^{13} \mathrm{C}$ NMR $\delta 142.4,140.0,132.5,131.1,125.8,123.3,121.0,36.1,31.9,31.7,29.62,29.59,29.5$ 29.34, 29.31, 22.6, 14.1; MS (EI) m/z $520\left(\mathrm{M}^{+}\right)$; Anal. Calcd for $\mathrm{C}_{34} \mathrm{H}_{48} \mathrm{~S}_{2}$ : C, 78.40; H, 9.29. Found: C, 78.36; H, 9.29.

\section{2,7-Diundecyl[1]benzothieno[3,2-b][1]benzothiophene $(\mathrm{n}=11)$ :}

$89 \%$ yield. mp $125-127{ }^{\circ} \mathrm{C} ;{ }^{1} \mathrm{H}$ NMR $\delta 7.75(\mathrm{~d}, J=8.1 \mathrm{~Hz}, 2 \mathrm{H}), 7.69$ (s, 2H), 7.26 (d, J=8.1 Hz, 2H), $2.74(\mathrm{t}, J=7.7 \mathrm{~Hz}, 4 \mathrm{H}), 1.72-1.65(\mathrm{~m}, 4 \mathrm{H}), 1.33-1.26(\mathrm{~m}, 32 \mathrm{H}), 0.87(\mathrm{t}, J=6.8 \mathrm{~Hz}, 6 \mathrm{H}) ;{ }^{13} \mathrm{C}$ NMR $\delta 142.4,140.0,132.5,131.1,125.8,123.3,121.0,36.1,31.9,31.7,29.7,29.63,29.60,29.5$ 29.4, 29.3, 22.6, 14.1; MS (EI) $\mathrm{m} / \mathrm{z} 548\left(\mathrm{M}^{+}\right)$; Anal. Calcd for $\mathrm{C}_{36} \mathrm{H}_{52} \mathrm{~S}_{2}$ : C, 78.77; H, 9.55. Found: C, 78.75; H, 9.44.

2,7-Didodecyl[1]benzothieno[3,2-b][1]benzothiophene $(\mathbf{n}=12)$ :

$80 \%$ yield. mp $121-123{ }^{\circ} \mathrm{C} ;{ }^{1} \mathrm{H}$ NMR $\delta 7.77(\mathrm{~d}, J=8.3 \mathrm{~Hz}, 2 \mathrm{H}), 7.70(\mathrm{~d}, J=1.2 \mathrm{~Hz}, 2 \mathrm{H}), 7.27$ (dd, $J=1.2,8.3 \mathrm{~Hz}, 2 \mathrm{H}), 2.75(\mathrm{t}, J=7.8 \mathrm{~Hz}, 4 \mathrm{H}), 1.72-1.65(\mathrm{~m}, 4 \mathrm{H}), 1.34-1.25(\mathrm{~m}, 36 \mathrm{H}), 0.87(\mathrm{t}, J=$ $6.8 \mathrm{~Hz}, 6 \mathrm{H}) ;{ }^{13} \mathrm{C}$ NMR $\delta 142.4,140.0,132.5,131.1,125.8,123.3,121.0,36.1,31.9,31.7,29.65$, 29.65, 29.63, 29.58, 29.51, 29.35, 29.29, 22.7, 14.1; MS (EI) m/z $576\left(\mathrm{M}^{+}\right)$; Anal. Calcd for $\mathrm{C}_{38} \mathrm{H}_{56} \mathrm{~S}_{2}:$ C, 79.10; H, 9.78. Found: C, 79.19; H, 9.85

\section{2,7-Ditridecyl[1]benzothieno[3,2-b][1]benzothiophene $(\mathbf{n}=13)$ :}

$80 \%$ yield. mp $120-122{ }^{\circ} \mathrm{C} ;{ }^{1} \mathrm{H}$ NMR $\delta 7.76(\mathrm{~d}, J=8.1 \mathrm{~Hz}, 2 \mathrm{H}), 7.70(\mathrm{~s}, 2 \mathrm{H}), 7.27(\mathrm{~d}, J=8.1 \mathrm{~Hz}$, 2H), $2.75(\mathrm{t}, J=7.7 \mathrm{~Hz}, 4 \mathrm{H}), 1.73-1.65(\mathrm{~m}, 4 \mathrm{H}), 1.34-1.25(\mathrm{~m}, 40 \mathrm{H}), 0.88(\mathrm{t}, J=6.8 \mathrm{~Hz}, 6 \mathrm{H}) ;{ }^{13} \mathrm{C}$ NMR $\delta 142.4,140.0,132.5,131.1,125.8,123.3,121.0,36.1,31.9,31.7,29.66(\times 4), 29.59,29.52$, 29.36, 29.30, 22.7, 14.1; MS (EI) m/z $604\left(\mathrm{M}^{+}\right)$; Anal. Calcd for $\mathrm{C}_{40} \mathrm{H}_{60} \mathrm{~S}_{2}$ : C, 79.40; H, 10.00 . Found: C, 79.56; H, 10.06 .

\section{2,7-Ditetradecyl[1]benzothieno[3,2-b][1]benzothiophene $(\mathbf{n}=14)$ :}

Recrystallization from hexane-chloroform; 91\% yield. mp $117-118{ }^{\circ} \mathrm{C} ;{ }^{1} \mathrm{H}$ NMR $\delta 7.76(\mathrm{~d}, J=8.1$ $\mathrm{Hz}, 2 \mathrm{H}), 7.70(\mathrm{~d}, J=1.4 \mathrm{~Hz}, 2 \mathrm{H}), 7.27(\mathrm{dd}, J=1.4,8.1 \mathrm{~Hz}, 2 \mathrm{H}), 2.75(\mathrm{t}, J=7.6 \mathrm{~Hz}, 4 \mathrm{H})$, $1.72-1.65(\mathrm{~m}, 4 \mathrm{H}), 1.34-1.25(\mathrm{~m}, 44 \mathrm{H}), 0.88(\mathrm{t}, J=6.8 \mathrm{~Hz}, 6 \mathrm{H}) ;{ }^{13} \mathrm{C}$ NMR $\delta 142.3,140.0,132.5$, 131.1, 125.8, 123.3, 121.0, 36.1, 31.9, 31.7, $29.66(\times 5), 29.58,29.51,29.36,29.30,22.7,14.1$; MS (EI) $\mathrm{m} / \mathrm{z} 632\left(\mathrm{M}^{+}\right)$; Anal. Calcd for $\mathrm{C}_{42} \mathrm{H}_{64} \mathrm{~S}_{2}$ : C, 79.68; H, 10.19. Found: C, 79.71; H, 10.22.

The products obtained by the two synthetic methods were identical (NMR, mp, UV-vis etc.) and the FET devices based on both samples showed almost the same results within the experimental error. Therefore, the samples obtained via both routes can utilized for device fabrication, provided that they are sufficiently purified by chromatography and recrystallization.

From the synthetic chemical point of view, however, Rout 2 in Scheme S1 is advantageous, because various acetylene derivatives are readily available and easy to handle than the acid chloride. In addition, Route 1 can not be applicable to the synthesis of ditetradecyl derivative ( $\mathrm{n}=$ 14), owing to poor reactivity of the acid chloride with the parent BTBT. Therefore, most of the 
FET works were done with the sample synthesized via Route 2. On the other hand, for the synthesis of large quantity of the sample of derivatives with $\mathrm{n}=8,10,12$, Route 1 is advantageous.

\section{Physicochemical properties}

Methods: UV-vis spectra in dichloromethane solution were recorded on a Shimadzu UV-3100 spectrometer. Differential scanning calorimetry was carried out on a Seiko Instruments EXSTAR6000 DSC6200. Cyclic voltammograms (CVs) were recorded on a Hokuto Denko HA-301 potentiostat and a Hokuto Denko HB-104 function generator in benzonitrile containing tetrabutylammonium hexafluorophosphate $\left(\mathrm{Bu}_{4} \mathrm{NPF}_{6}, 0.1 \mathrm{M}\right)$ as supporting electrolyte at a scan rate of $100 \mathrm{mV} / \mathrm{s}$. Counter and working electrodes were made of $\mathrm{Pt}$, and the reference electrode was $\mathrm{Ag} / \mathrm{AgCl}$. All the potentials were calibrated with the standard ferrocene/ferrocenium redox couple $\left(\mathrm{E}^{1 / 2}=+0.46 \mathrm{~V}\right.$ measured under identical conditions).

All the derivatives of $C_{n}$-BTBT $(n=5 \sim 14)$ showed almost identical UV-vis spectra and cyclic voltammograms, and thus, those of $\mathrm{C}_{12}$-BTBT were shown in Figure $\mathrm{S} 1$ as representatives. Their HOMO energy levels were estimated from the on-set of oxidation peak in the voltammograms to be ca $5.5 \mathrm{eV}$ below the vacuum level. ${ }^{\mathrm{S} 3}$ The HOMO-LUMO gap estimated from the absorption edge in the UV-vis spectra was $3.5 \mathrm{eV}$, which gave the LUMO level of $2.0 \mathrm{eV}$ below the vacuum level.

(a)

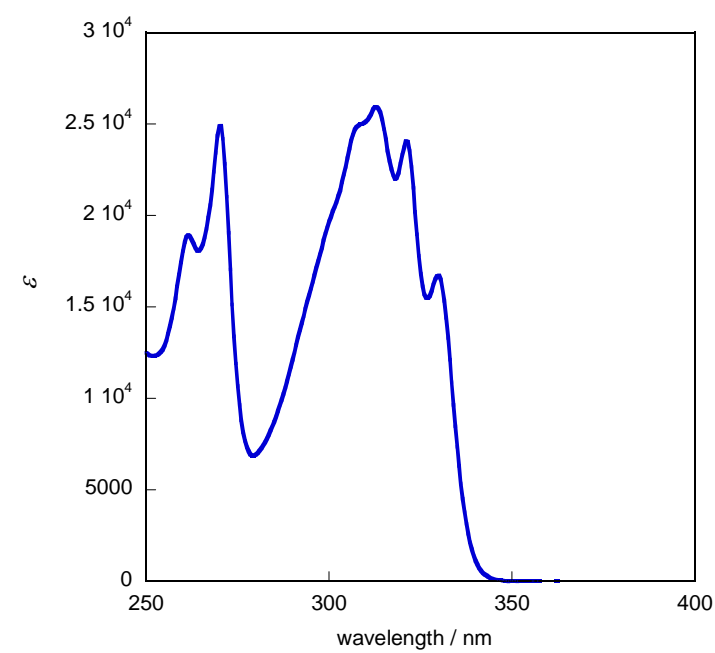

(b)

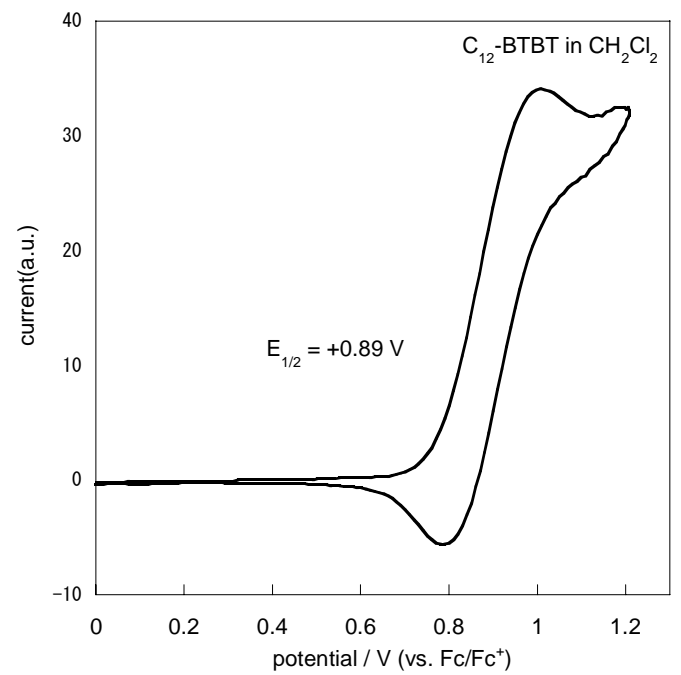

Figure S1. (a) UV-Vis spectra of $\mathrm{C}_{12}$-BTBT in chloroform solution. UV-Vis spectra of other $\mathrm{C}_{n}$-BTBTs were essentially the same with those for $\mathrm{C}_{12}$-BTBT; (b) Cyclic voltammogram of $\mathrm{C}_{12}$-BTBT. 
Table S1. List of thermal transition temperatures

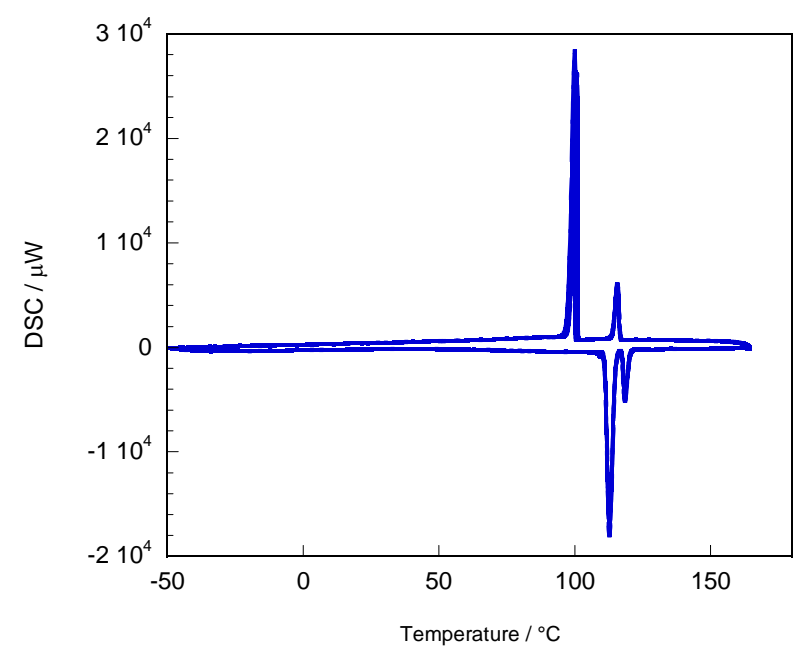

on DSC analysis and solubility of $\mathrm{C}_{n}$-BTBTs.

\begin{tabular}{cccc}
\hline$n$ & $\begin{array}{c}\mathrm{mp} /{ }^{\circ} \mathrm{C}(\text { on } \\
\text { hot plate })\end{array}$ & $T_{\mathrm{C} \rightarrow \mathrm{LC}} /{ }^{\circ} \mathrm{C}$ & $\begin{array}{c}\mathrm{T}_{\mathrm{LC} \rightarrow \mathrm{I}} \\
/{ }^{\circ} \mathrm{C}\end{array}$ \\
\hline 5 & $121-122$ & - & 121.8 \\
6 & $122-124$ & 115.8 & 119.9 \\
7 & $129-132$ & 109.3 & 129.5 \\
8 & $129-131$ & 111.2 & 126.5 \\
9 & $129-131$ & 106.7 & 127.8 \\
10 & $124-126$ & 112.2 & 124.2 \\
11 & $125-127$ & 109.2 & 122.9 \\
12 & $121-123$ & 112.7 & 118.3 \\
13 & $120-122$ & 112.3 & 116.7 \\
14 & $117-118$ & - & 114.3 \\
\hline
\end{tabular}

C: crystal phase, LC: liquid crystal phase, I: isotropic liquid

As described in reference $\mathrm{S} 1$, the endothermic peaks at around $110{ }^{\circ} \mathrm{C}$ correspond to the crystal-liquid-crystal (LC) phase transition. The LC phase was reported to be the SmA phase. ${ }^{\mathrm{S} 1} \mathrm{By}$ comparing the FET characteristics of the derivatives, the phase transition temperatures were not affective to the performances of devices. However, we noticed that annealing of the spin-coated thin films at the high temperature than $T_{\mathrm{C} \rightarrow \mathrm{LC}}$ or $T_{\mathrm{LC} \rightarrow \mathrm{I}}$ reduce the device characteristics. Annealing at higher temperature may cause the formation of discontinuous crystal grains, that deters the semiconducting channel in the FET devices.

\section{Solubility and stability}

Solubility determination: To a precisely weighed sample (ca. 5 or $10 \mathrm{mg}$ ), chloroform was added in increments of $0.1 \mathrm{~mL}$, and the resulting mixture was shaken and sonicated at room temperature $\left(20{ }^{\circ} \mathrm{C}-25^{\circ} \mathrm{C}\right)$. The total amount of solvent required to dissolve the entire solid sample was converted into solubility $\left(\mathrm{g} \mathrm{L}^{-1}\right)$. The results are listed in Table 1 in the main text.

\section{Chemical Stability of $\mathrm{C}_{12}$-BTBT in solution}

Solution of $\mathrm{C}_{12}$-BTBT in air-saturated

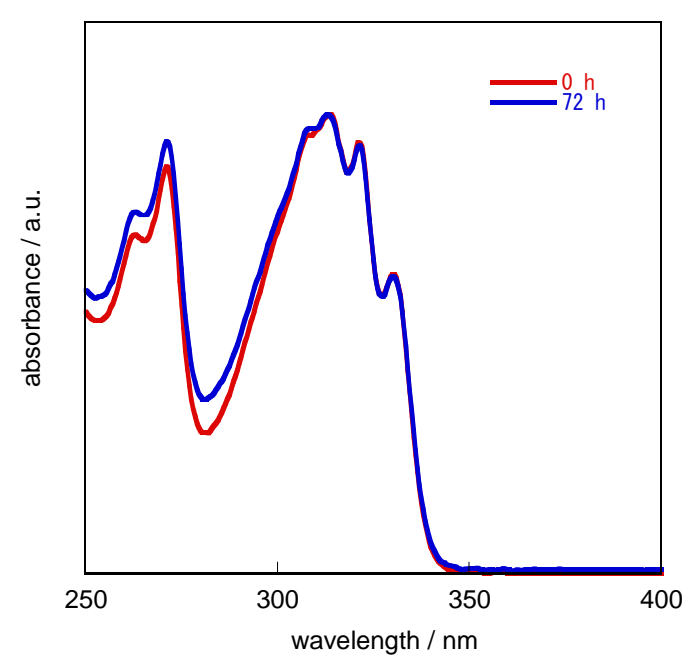

Figure S3. UV-Vis spectra of $\mathrm{C}_{12}$-BTBT in air-saturated dichloromethane solution. 
dichloromethane was prepared, and its UV-vis spectra were recorded. The UV-vis spectra were virtually no changed even after $72 \mathrm{~h}$ under ambient atmosphere in dark as shown in Figure S3. Pentacene, on the other hand, was rapidly degraded under the same experimental conditions with in $24 \mathrm{~h}$ as already reported. ${ }^{\mathrm{S}}$

\section{Single crystal $X$-ray analysis of $\mathrm{C}_{12}$-BTBT}

Single crystals suitable for structural analysis were obtained by careful recrystallization from hexane. The X-ray crystal structure analysis was made on a Rigaku Mercury-CCD (MoK $\alpha$ radiation, $\lambda=0.71069 \AA$, graphite monochromator, $T=296 \mathrm{~K}, 2 \theta_{\max }=55.0^{\circ}$ ). The structure was solved by the direct methods. ${ }^{\mathrm{S} 5}$ Non-hydrogen atoms were refined anisotropically, and hydrogen atoms were included in the calculations but not refined. All calculations were performed using the crystallographic software package TeXsan 1.2. ${ }^{\mathrm{S} 6}$

Crystal data for $\mathrm{C}_{12}$-BTBT: $\mathrm{C}_{38} \mathrm{H}_{56} \mathrm{~S}_{2}, M=576.98$, colorless plate, $0.60 \times 0.40 \times 0.10 \mathrm{~mm}^{3}$, Monoclinic, space group, $P 2{ }_{1} / a, a=5.864(5), b=7.740(7), c=37.91(3) \AA, \beta=90.589(3){ }^{\circ}, \mathrm{V}=$ 1721(3) $\AA^{3}, Z=2, R=0.0608$ for 1651 observed reflections $(\mathrm{I}>2 \sigma(\mathrm{I}))$ and 182 variable parameters, $w R^{2}=0.1646$ for all data (3497).

(a)

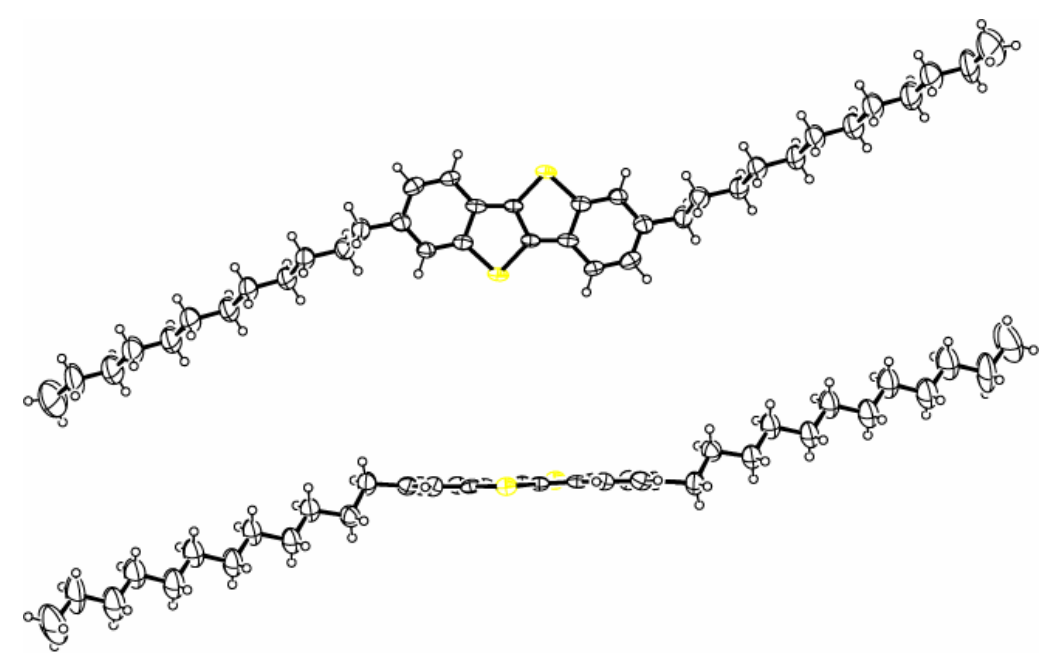

Figure S4. Molecular structure of $\mathrm{C}_{12}$-BTBT: (a) top view; (b) side view.

\section{Device fabrications and evaluations}

OFET devices were fabricated in a "top-contact" configuration on a heavily doped $n^{+}-\mathrm{Si}(100)$ wafer with 200-nm-thick thermally grown $\mathrm{SiO}_{2}\left(C_{i}=17.3 \mathrm{nF} \mathrm{cm}{ }^{-2}\right.$, without surface modification). A thin film (ca. $80 \sim 100 \mathrm{~nm}$ thick) of $\mathrm{C}_{n}$-BTBT as the active layer was deposited on the $\mathrm{Si} / \mathrm{SiO}_{2}$ substrates by spin coating a $0.4 \mathrm{wt} \%$ solution of the $\mathrm{C}_{n}$-BTBT in chloroform at $4000 \mathrm{rpm}$ for 30 sec, and dried at $80^{\circ} \mathrm{C}$ on a hot-plate for 30 min under nitrogen atmosphere. On top of the organic thin film, gold films $(80 \mathrm{~nm})$ as drain and source electrodes were deposited through a shadow 
mask. For a typical device, the drain-source channel length $(L)$ and width $(W)$ are $50 \mu \mathrm{m}$ and ca. $1.5 \mathrm{~mm}$, respectively. The devices were then annealed at $80{ }^{\circ} \mathrm{C}$ for $30 \mathrm{~min}$ under nitrogen atmosphere. The characteristics of the OFET devices were evaluated at room temperature in air with a Keithly 6430 subfemtoampmeter and a Keithly 2400 source meter, operated by a LabTracer program and GPIB interface. Field-effect mobility $\left(\mu_{\mathrm{FET}}\right)$ was calculated in the saturation regime $\left(V_{d}=-60 \mathrm{~V}\right)$ of the $I_{\mathrm{d}}$ using the following equation,

$I_{\mathrm{d}}=\left(W C_{\mathrm{i}} / 2 L\right) \mu_{\mathrm{FET}}\left(V_{\mathrm{g}}-V_{\mathrm{th}}\right)^{2}$

where $C_{i}$ is the capacitance of the $\mathrm{SiO}_{2}$ insulator, and $V_{\mathrm{g}}$ and $V_{\text {th }}$ are the gate and threshold voltages, respectively. Current on/off ratio $\left(I_{\mathrm{on}} / I_{\mathrm{off}}\right)$ was determined from the $I_{\mathrm{d}}$ at $V_{\mathrm{g}}=0 \mathrm{~V}\left(I_{\mathrm{off}}\right)$ and $V_{\mathrm{g}}=-60$ $\mathrm{V}\left(I_{\mathrm{on}}\right)$. The $\mu_{\mathrm{FET}}$ data reported in Table 1 are typical vales from more than 20 different devices.

The shelf lifetime tests were carried out as follows: OFET devices were stored in a plastic box, and the FET characteristics were measured at 20, 110, and 160 days after fabrication of the devices.

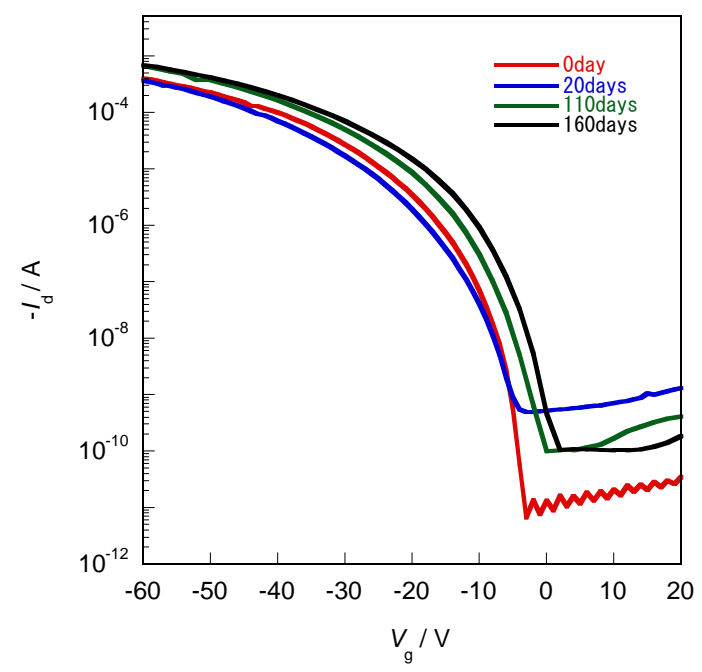

Figure S5. Transfer characteristics of $\mathrm{C}_{12}$-BTBT-based devices measured after 20, 110, 160 days after fabrication of devices.

\section{XRDs of $\mathrm{C}_{n}$-BTBT thin film}

X-ray diffractions of spin-coated thin films deposited on bare $\mathrm{Si} / \mathrm{SiO}_{2}$ substrates were obtained with a PANalytical X'Port PRO MPD diffractometer with a $\mathrm{Cu} K \alpha$ source $(\lambda=1.541 \AA)$ in air. Calculated interlayer distances ( $d$-spacings) are listed in Table 1 in the main text. Crystallite sizes estimated from (001) or (002) reflections with the Scherrer method are in the range of ca. 30 to 50 $\mathrm{nm}$ for all $\mathrm{C}_{n}$-BTBTs (Table S2). Therefore, we conclude that the effects of the alkyl chain length on the crystallite size and consequently on the FET characteristics are marginal. 
Table S2. Estimation of crystallite sizes using the out-of-plane-XRD data

$\mathrm{D}(\mathrm{nm})$ based on (001) reflections

\begin{tabular}{|c|c|c|c|c|c|c|c|c|c|c|}
\hline $\mathrm{R}$ & $\mathrm{C} 5$ & $\mathrm{C} 6$ & $\mathrm{C} 7$ & $\mathrm{C} 8$ & $\mathrm{C} 9$ & $\mathrm{C} 10$ & $\mathrm{C} 11$ & $\mathrm{C} 12$ & $\mathrm{C} 13$ & $\mathrm{C} 14$ \\
\hline $2 \theta /{ }^{\circ}$ & 3.878 & 3.605 & 3.239 & 3.052 & 2.780 & 2.644 & 2.457 & 2.36 & 2.202 & 2.097 \\
\hline$\theta /{ }^{\circ}$ & 1.939 & 1.802 & 1.670 & 1.526 & 1.390 & 1.322 & 1.228 & 1.180 & 1.101 & 1.048 \\
\hline $\cos \theta$ & 0.999 & 0.999 & 0.999 & 0.999 & 0.999 & 0.999 & 0.999 & 0.999 & 0.999 & 0.999 \\
\hline$\beta /$ radian & 0.00375 & 0.00340 & 0.00463 & 0.00436 & 0.00419 & 0.00384 & 0.00405 & 0.00379 & 0.00349 & 0.00356 \\
\hline$D / \mathrm{nm}$ & 43.8 & 48.7 & 35.8 & 37.8 & 39.4 & 43.0 & 40.8 & 43.4 & 47.3 & 41.3 \\
\hline
\end{tabular}

$\mathrm{D}(\mathrm{nm})$ based on (002) reflections

\begin{tabular}{|c|c|c|c|c|c|c|c|c|c|c|}
\hline $\mathrm{R}$ & $\mathrm{C} 5$ & $\mathrm{C} 6$ & $\mathrm{C} 7$ & $\mathrm{C} 8$ & $\mathrm{C} 9$ & $\mathrm{C} 10$ & $\mathrm{C} 11$ & $\mathrm{C} 12$ & $\mathrm{C} 13$ & $\mathrm{C} 14$ \\
\hline $2 \theta /{ }^{\circ}$ & 7.710 & 7.200 & 6.520 & 6.129 & 5.653 & 5.347 & 4.973 & 4.718 & 4.463 & 4.273 \\
\hline$\theta /{ }^{\circ}$ & 3.855 & 3.600 & 3.260 & 3.065 & 2.827 & 2.674 & 2.487 & 2.359 & 2.231 & 2.136 \\
\hline $\cos \theta$ & 0.998 & 0.998 & 0.998 & 0.999 & 0.999 & 0.999 & 0.999 & 0.999 & 0.999 & 0.999 \\
\hline$\beta /$ radian & 0.00377 & 0.00384 & 0.00524 & 0.00419 & 0.00410 & 0.00532 & 0.00543 & 0.00445 & 0.00583 & 0.00569 \\
\hline$D / \mathrm{nm}$ & 43.8 & 43.0 & 31.5 & 39.4 & 40.1 & 30.9 & 30.3 & 36.9 & 28.4 & 29.0 \\
\hline
\end{tabular}

Scherrer equation:

$D=K \cdot \lambda / \beta \cos \theta$

where

$K$ : Scherrer constant (1.07 for sphere shaped crystallite)

$\lambda$ : wave length of X-ray used (1.5045 $\AA$ )

$\theta$ : Bragg angle from $2 \theta$ profile

$\beta$ : full width of half maximum intensity (FWHM) expressed by radian

Collection of $\beta$ was not carried out because of the lack of suitable standard sample in such low $2 \theta$ angle region. Therefore, the values listed here are not suitable for quantitative discussion, but the results indicate that the crystallite sizes of different compounds are in a similar range.

\section{In-plane XRD of $\mathrm{C}_{12}$-BTBT thin film}

In-plane X-ray diffractions of the thin film of $\mathrm{C}_{12}$-BTBTs spun on the $\mathrm{Si} / \mathrm{SiO}_{2}$ substrates were measured with a Rigaku SmartLab with a $\mathrm{Cu} K \alpha$ source $(\lambda=1.541 \AA)$ in air. Figure S6(a) shows $2 \theta \chi / \phi$ scan profiles with different incident angles $\left(0.19,0.22\right.$, and $\left.0.25^{\circ}\right)$. With large incident angles, halos originating from the substrate $\left(\mathrm{Si} / \mathrm{SiO}_{2}\right)$ are observed. All three peaks are assignable with the structural data of the single crystal X-ray analysis of $\mathrm{C}_{12}-\mathrm{BTBT}$ : for comparison, the simulated powder pattern based on the single crystal data is shown in Figure S6(b). Crystallite size estimated from the (020) reflection with the Scherrer method is ca. $40 \mathrm{~nm}$. 
(a)

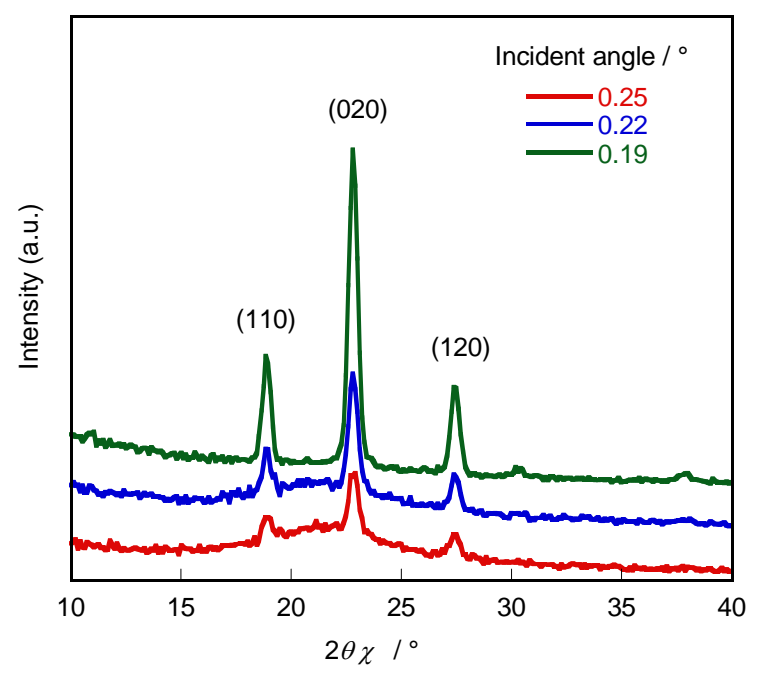

(b)

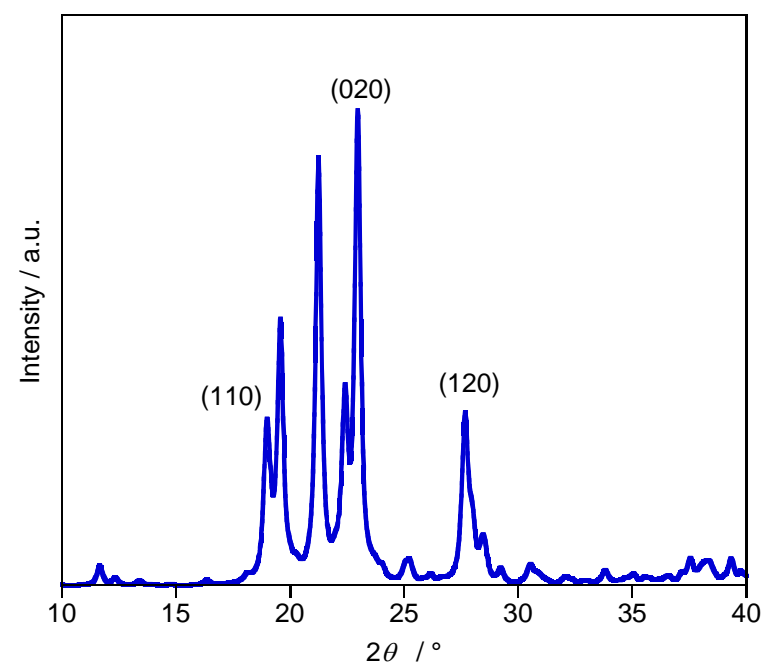

Figure S6. (a) $2 \theta \chi / \phi$ Scan profiles of $\mathrm{C}_{12}$-BTBT thin film with different incident angles. (b) A powder pattern simulated based on the single crystal data of $\mathrm{C}_{12}$-BTBT.

Figure S7 shows a $\phi$ scan profile of (020) reflection. No apparent angle dependence indicates the random orientation of the crystallite in the thin film. The small intensity distribution may be caused by the different shape of the crystallites.

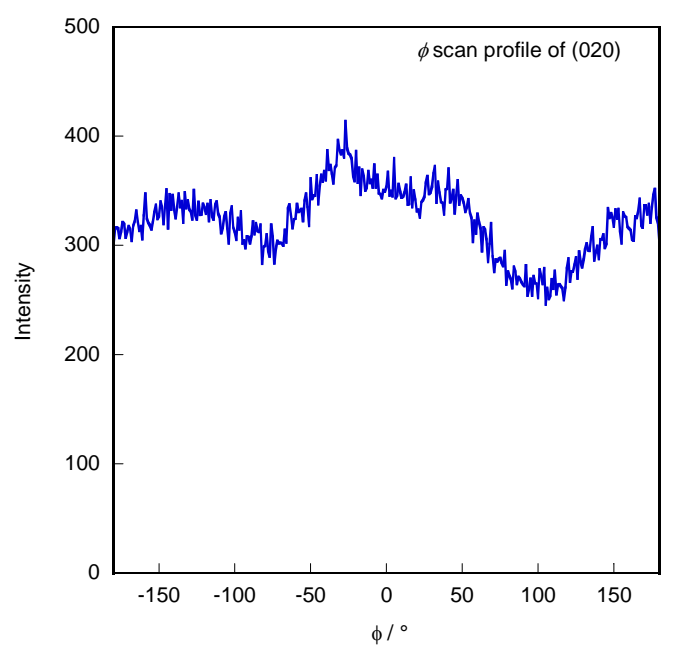

Figure S7. $\phi$ Scan profile of $(020)$ reflection.

\section{References}

S1. Košata, B.; Kozmik, V.; Svoboda, J.; Novotaná, Vanák, P.; Glogarvá, M., Liq. Cryst. 2003, 30, 603-610.

S2. Takimiya, K.; Ebata, H.; Sakamoto, K.; Izawa, T.; Otsubo, T.; Kunugi, Y. J. Am. Chem. Soc. 2006, 128, 12604-12605.

S3. (a) Brédas, J.-L.; Silbey, R.; Boudreaux, D. S.; Chance, R. R. J. Am. Chem. Soc. 1983, 105, 6555-6559. (b) Pommerehne, J.; Vestweber, H.; Guss, W.; Mark, R. F.; Bässler, H.; Porsch, M.; Daub, J. Adv. Mater. 1995, $7,551-554$.

S4. Maliakal, A.; Raghavachari, K.; Katz, H.; Chandross, E.; Siegrist, T. Chem. Mater. 2004, 16, 4980-4986. 
S5. Sir92 Altomare A.; Cascarano G.; Giacovazzo C.; Guagliardi A.; Burla M. C.; Polidori G.; Camalli M. J. Appl. Cryst. 1994, 27, 435-435.

S6. teXsan: Single Crystal Structure Analysis Software, Version 1.19; Molecular Structure Corporation and Rigaku Corporation, 2000. 